\title{
Influence of phase separation on the linear viscoelastic behavior of a miscible polymer blend
}

\author{
Suresh Mani, Michael F. Malone, \\ and $\mathrm{H}$. Henning Winter ${ }^{\mathrm{a})}$ \\ Chemical Engineering Department, University of \\ Massachusetts, Amherst, Massachusetts 01003-0011
}

(Received 20 December 1991; accepted 1 June 1992)

\section{Synopsis}

The influence of phase separation on the linear viscoelastic response has been studied in miscible blends of polystyrene and poly (vinyl methyl ether) with a lower critical solution temperature near $110^{\circ} \mathrm{C}$. At temperatures between 25 and $155^{\circ} \mathrm{C}$, and for compositions in the range $20 \%$ to $60 \%$ polystyrene, the complex moduli $G^{\prime}$ and $G^{\prime \prime}$ were measured at frequencies in the range of 0.01 to $100 \mathrm{rad} / \mathrm{s}$. Time-temperature superposition was applied in the single phase region to obtain the complex modulus over eight decades of frequency. Increasing the polystyrene content resulted in an increase in the zero-shear viscosity and a shift of the terminal behavior to lower frequencies or longer times. The phase separation above the lower critical solution temperature was measured as a sudden increase in the fluorescence intensity of an anthracene-labeled polystyrene (approximately $1 \mathrm{wt} \%$ in the blend), using an optical probe in the rheometer fixture. For the 20/80 and 40/60 PS/PVME samples, the terminal zone was in the accessible frequency window and phase separation was accompanied with a large increase in $G^{\prime}$ and $G^{\prime \prime}$. In contrast, the complex modulus of the 60/40 PS/PVME blend could not be measured near the terminal zone and the $G^{\prime}$ and $G^{\prime \prime}$ did not exhibit any significant changes near the phase transition temperature.

\section{INTRODUCTION}

For nearly two decades, there has been an increasing interest in finding new miscible polymer blends and understanding their properties. Many miscible polymer blends have been prepared by exploiting

a) To whom correspondence should be sent. 
specific interactions such as hydrogen bonding between polar groups attached to the polymer chains (Paul, 1978). Recently, the rheological characterization of miscible polymer blends and the influence of phase separation on the viscoelastic properties are receiving more attention.

A blend of polystyrene (PS) and poly (2,6-dimethyl phenylene oxide) (PPE) which is miscible over a wide composition range and at temperatures as high as $290^{\circ} \mathrm{C}$, was one of the first miscible blends to be developed. The dynamic mechanical response of these PS/PPE blends (the molecular weights of the components were approximately the same) at various compositions was compared using an "iso-free volume" state (Prest and Porter, 1972). The free volume of the blend was taken as the sum of the free volumes of the components weighted by their volume fractions, and the temperature was adjusted in order to keep this free volume constant over the composition range. Increasing amounts of PPE resulted in an increased viscosity and modulus, and a shift in the terminal region to longer times; more recent studies by Araujo and Stadler (1988) of the same blend system, with a ratio of molecular weights, $\boldsymbol{M}_{\mathrm{PS}} / \boldsymbol{M}_{\mathrm{PPE}} \cong 100$, showed PPE to behave as a low molecular weight diluent.

Another miscible blend system which has received considerable study is the polystyrene/poly (vinyl methyl ether) blend. In the single phase region, the addition of PVME plasticizes PS (Yang et al., 1986; Schneider and Brekner, 1985), which can be seen in the shifting of the $G^{\prime}, G^{\prime \prime}$ terminal zone to higher frequencies as the PVME content is increased (Brekner et al, 1985). However, close to the glass transition temperature (approximately $T_{g}+5 \mathrm{~K}$ ), complicated microstructural changes are suggested by a failure of time-temperature superposition (Cavaille et al., 1987).

Stadler et al. (1988) measured the complex modulus of a PS/PVME blend with low molecular weight PVME $\left(M_{\mathrm{PS}} / M_{\mathrm{PVME}} \cong 40\right)$, and found separate relaxations for PVME and PS in the entanglement region. This is typically seen in the one-phase region of miscible binary polymer blends, where the pure components have very different relaxation times. Surprisingly, phase separation was reported to have little effect on the relaxation behavior and time-temperature superposition was applied to $G^{\prime}, G^{\prime \prime}$ data measured as much as $40 \mathrm{~K}$ above the LCST. Only at higher temperatures did superposition break down due to a decrease in $G^{\prime}$ and, at about $90 \mathrm{~K}$ above the LCST, the dynamic moduli exhibited a power law behavior in frequency with an exponent of 0.6.

Time-temperature superposition was applied in the single-phase region of another PS/PVME blend $\left(M_{\mathrm{PS}} / M_{\mathrm{PVME}} \cong 2\right)$ by Ajji $e t$ al. 
(1989), who reported that phase separation resulted in an increase in $G^{\prime}$ but did not affect $G^{\prime \prime}$, which seems to be inconsistent with the KramersKronig relation (e.g., Bird et al., 1977). They also suggest that a plot of $\eta^{\prime} / \eta_{0}$ versus $\eta^{\prime \prime} / \eta_{0}$ (Cole-Cole plot) is more sensitive to the phase separation than the frequency dependence of $G^{\prime}$ and $G^{\prime \prime}$. Subsequently, Ajii and Choplin (1991) performed a scaling analysis using Frederickson and Larson's (1987) mean-field expression for the contribution of critical fluctuations to the shear stress in binary homopolymer blends. Their scaling analysis suggested that the fluctuations contributed less to $G^{\prime \prime}$ than to $G^{\prime}$.

In summary, for both PS/PPE and PS/PVME blend systems, in dynamic mechanical measurements, the transition to the terminal behavior is more gradual in the blend than for the pure components suggesting strong inter molecular interactions. The coexistence temperature in PS/PVME blends is much above the $T_{g}$ especially at low PS content. Time-temperature superposition can describe dynamic mechanical measurements at temperatures below the LCST down to approximately $10 \mathrm{~K}$ above the glass transition temperature. Previous studies show that phase separation does not have a measurable influence on the high-frequency behavior. The low-frequency behavior is more sensitive but this is not well understood. One report by Stadler et al. (1988) suggests that the complex modulus is not sensitive to phase separation, but Ajii et al. (1989) suggest that phase separation results in an increase in $G^{\prime}$ only. Clearly there is a need for a more extensive study to understand the effects of phase separation on the low-frequency behavior in miscible polymer blends.

The glassy motions of molecules in polymer blends can be monitored with deuterium NMR (e.g., Fischer et al., 1985; Landry and Henrichs, 1989). These motions contribute to the solid state properties such as impact strength and ductility. Mechanical spectroscopy does not seem to be a sensitive measure of this phenomenon.

The PS/PVME blend is a good model system, because its equilibrium phase behavior has been studied extensively and, unlike the PS/PPE blend, the LCST is experimentally accessible (the LCST occurs at about $110^{\circ} \mathrm{C}$ for the chosen PS/PVME blend). It is also known that large shear strains may raise the apparent LCST of PS/PVME blends (Mazich and Carr, 1983; Katsaros et al., 1989; Mani et al., 1991) by as much as $10 \mathrm{~K}$. This will be minimized by measuring within the linear viscoelastic range. In this study, we investigate the influence of phase separation on the linear viscoelastic behavior of a PS/PVME blend system as a function of temperature and composition. The fluorescence 
intensity of a blend containing labeled polystyrene is used to detect the onset of phase separation in a range of temperatures near the LCST and far above the glass transition.

\section{EXPERIMENTAL TECHNIQUES}

\section{Materials and sample preparation}

Polystyrene was obtained from Monsanto (Lustrex 101) with $M_{W}$ $=259 \mathrm{~kg} / \mathrm{mol}$ and $M_{W} / M_{N}=1.55$. Poly (vinyl methyl ether) was obtained from Scientific Polymer Products and has $M_{W}=85 \mathrm{~kg} / \mathrm{mol}$ and $M_{W} / M_{N}=1.39$. Labeled polystyrene, $\mathrm{PS}^{*}$ (chains of polystyrene with anthracene in the center), with a molecular weight of $200 \mathrm{~kg} / \mathrm{mol}$ was prepared according to the description given by Valeur et $a L$ (1974) and supplied to us by Dr. J. L. Halary and Professor L. Monnerie.

Blends were prepared with different compositions of PS/PVME and with 1 wt. \% of PS*, in toluene. Solvent-cast films were placed under a hood in a current of air, at room temperature, for approximately $48 \mathrm{~h}$ to remove most of the toluene and then held under vacuum at $70^{\circ} \mathrm{C}$ for 10 days to remove traces of moisture and toluene.

\section{Quiescent phase behavior}

The labeled polystyrene, PS*, is fluorescent in the visible region (approximately $440 \mathrm{~nm}$ ) when excited by photons in the ultraviolet region (approximately $365 \mathrm{~nm}$ ). However the fluorescence is substantially reduced when the PS* molecules are in close proximity (approximately 2 $\mathrm{nm}$ ) to the ether group of the PVME molecules. When a PS/PVME/ PS* sample is continuously excited, the fluorescence intensity emitted in the well-mixed state is low, but increases rapidly when the sample demixes. The phase transition temperatures from fluorescence measurements in PS/PVME blends agree well with small angle neutron scattering measurements (Halary et al., 1985) indicating that the addition of PS $^{*}$ does not affect the phase behavior. We have also found that the addition of PS* in trace amounts has no measurable influence on the rheology.

\section{Rheometer and rheometry procedure}

A Rheometrics RMS- 800 rheometer was recently modified for improved temperature control and in situ fluorescence measurements, as already described by Mani et al. (1991). As shown in Fig. 1, the sample is guarded between two isothermal regions (achieved by independently 


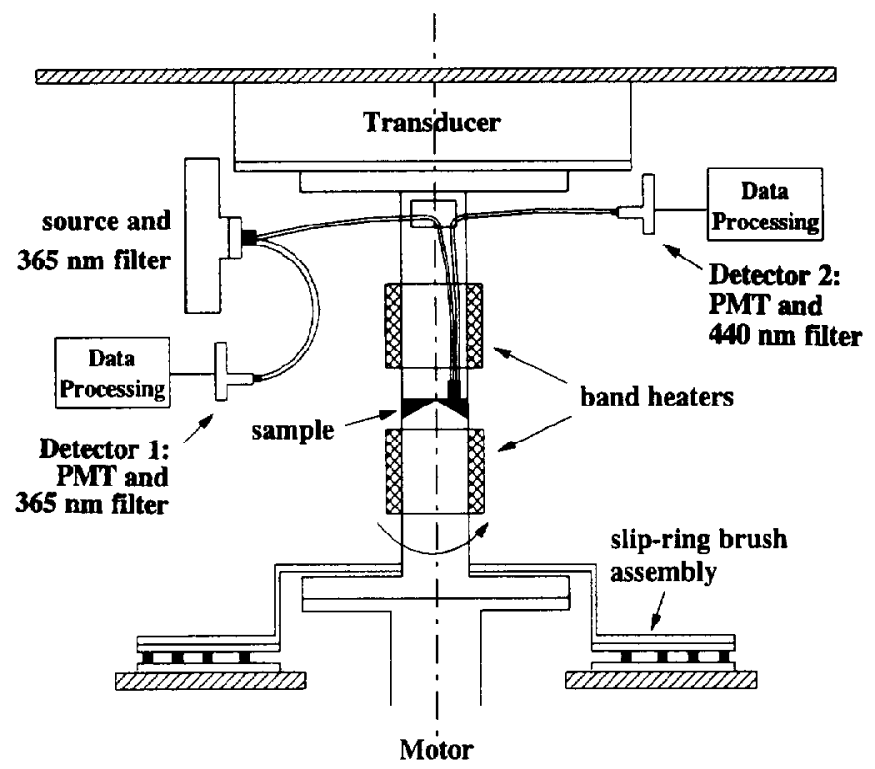

FIG. 1. Schematic of the modified rheometry equipment. The specially built fixtures for the rheometer (Rheometrics RMS-800), have band heaters mounted on them; each heater is independently controlled by a temperature controller. A rotating contact (slip-ring and brush assembly) is required for the thermocouple and the heater, which are mounted on the lower fixture. A bifurcated silica fiber optic probe is flush-mounted on the upper stationary plate for in situ fluorescence measurements; photons in the ultraviolet region excite the anthracene-labeled polystyrene in the sample and the fluorescence intensity is measured by a photomultiplier tube.

controlling the heaters mounted on each of the two fixtures), and thus eliminating thermal gradients. The complex modulus of the pure components polystyrene, poly (vinyl methyl ether), and the PS/PVME blends was measured in an oscillating parallel plate mode. Most experiments were performed with $25 \mathrm{~mm}$ diameter plates using a gap of 1 $\mathrm{mm}$. However, at higher temperatures and low frequencies, larger plates of $50 \mathrm{~mm}$ diameter were used to enhance the torque signal. The sample was protected by a dry nitrogen environment.

The $G^{\prime}$ and $G^{\prime \prime}$ of PS/PVME samples were measured at temperatures from 25 to $155^{\circ} \mathrm{C}$ and at compositions of 20/80, 40/60, and 60/40 (w/w) PS/PVME. To obtain reproducible results, the following protocol was developed: 


\section{0/60 PS/PVME}

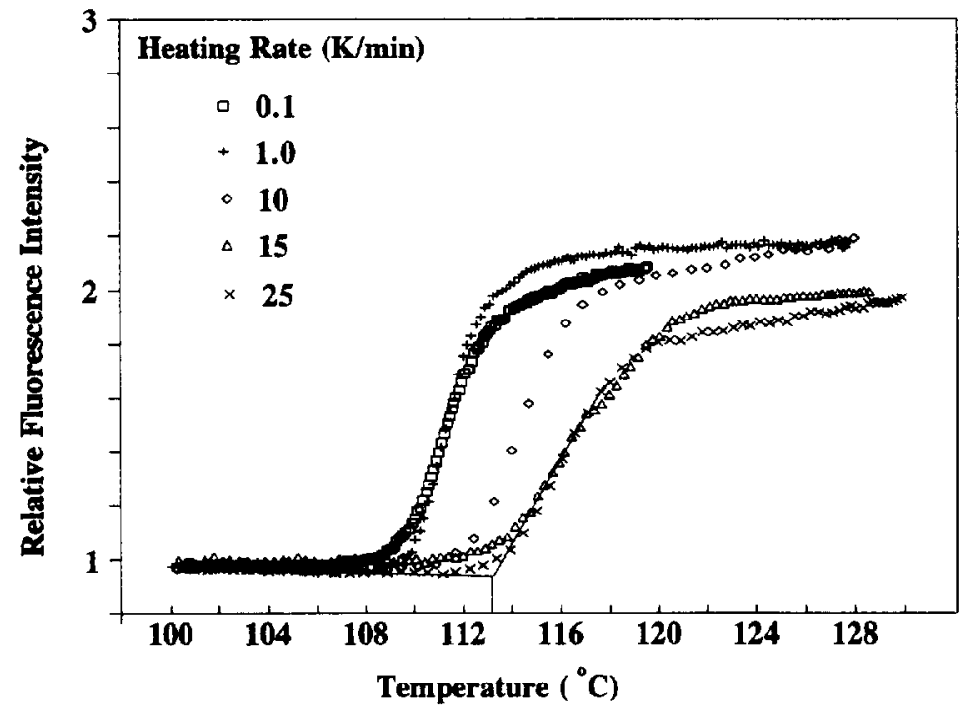

FIG. 2. The evolution of fluorescence intensity in a PS/PVME, $40 / 60$ blend at different heating rates ranging from 0.1 to $25 \mathrm{k} / \mathrm{min}$. (Fluorescence measurements are reported relative to the intensity of the mercury source; this relative intensity is normalized to unity at $90^{\circ} \mathrm{C}$.) The phase transition temperature was at $110^{\circ} \mathrm{C}$ for slow heating and at $113^{\circ} \mathrm{C}$ for fast heating.

(1) Heating at $10 \mathrm{~K} / \mathrm{min}$ brought the sample quickly to the desired temperature.

(2) A time sweep at $0.1 \mathrm{rad} / \mathrm{s}$ and a shear strain of 0.05 was performed until the $G^{\prime}$ and $G^{\prime \prime}$ values had become constant within the measurement error.

(3) Strain sweeps in the range from 0.05 to 1 shear strain, at frequencies of $0.1,1$, and $10 \mathrm{rad} / \mathrm{s}$ were used to determine the linear viscoelastic range for the material.

(4) Frequency sweeps in the range from 0.01 to $100 \mathrm{rad} / \mathrm{s}$ were made at strains in the linear viscoelastic range to obtain the $G^{\prime}, G^{\prime \prime}$ data.

For convenience, the dynamic mechanical data will be converted into the time domain (Baumgaertel and Winter, 1989). A discrete relaxation spectrum using small number of Maxwell modes is able to describe the data within experimental scatter. 


\section{PS/PYME}

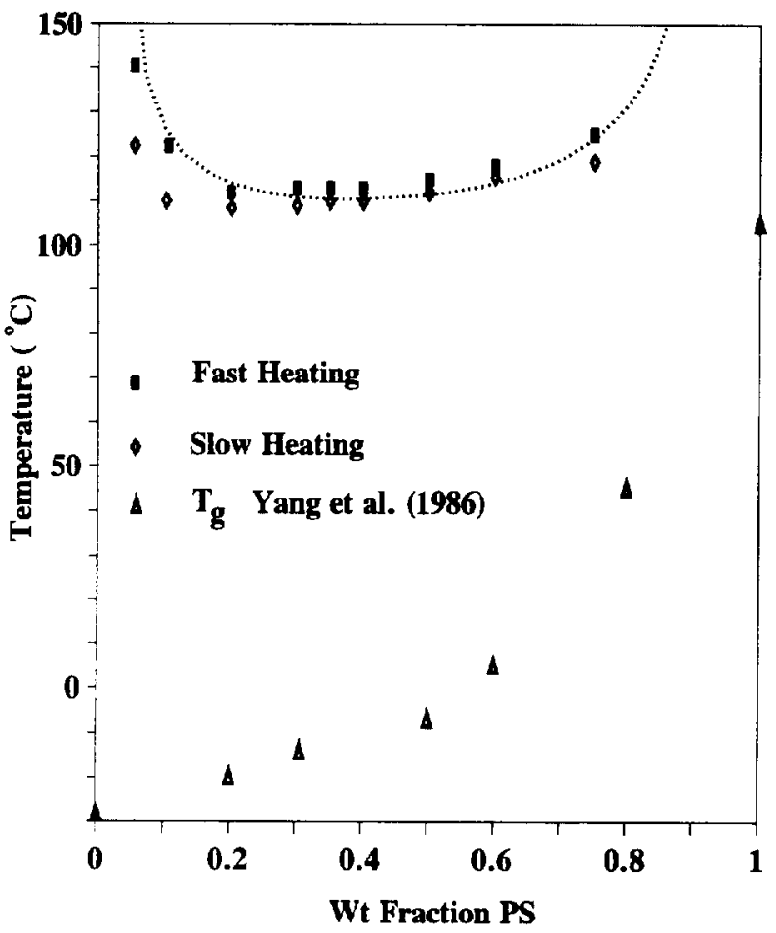

FIG. 3. The experimentally determined phase transition temperatures at slow and fast heating rates, are shown for different compositions. The glass transition temperature data were taken from Yang et al. (1986).

\section{RESULTS}

\section{Quiescent fluorescence measurements}

Samples were heated at rates of $0.1,1,10,15$, and $25 \mathrm{~K} / \mathrm{min}$. Two straight lines were drawn through the data for fluorescence intensity, as shown in Fig. 2 for the 40/60 PS/PVME sample. The temperature corresponding to the intersection is taken as the phase transition temperature; the reproducibility was $\pm 0.5 \mathrm{~K}$. "Slow" heating rates of 1 $\mathrm{K} / \mathrm{min}$ and lower gave the same phase transition temperature, while "fast" heating at $15 \mathrm{~K} / \mathrm{min}$ and higher gave a somewhat higher temperature. The measured phase transition temperatures are plotted in 


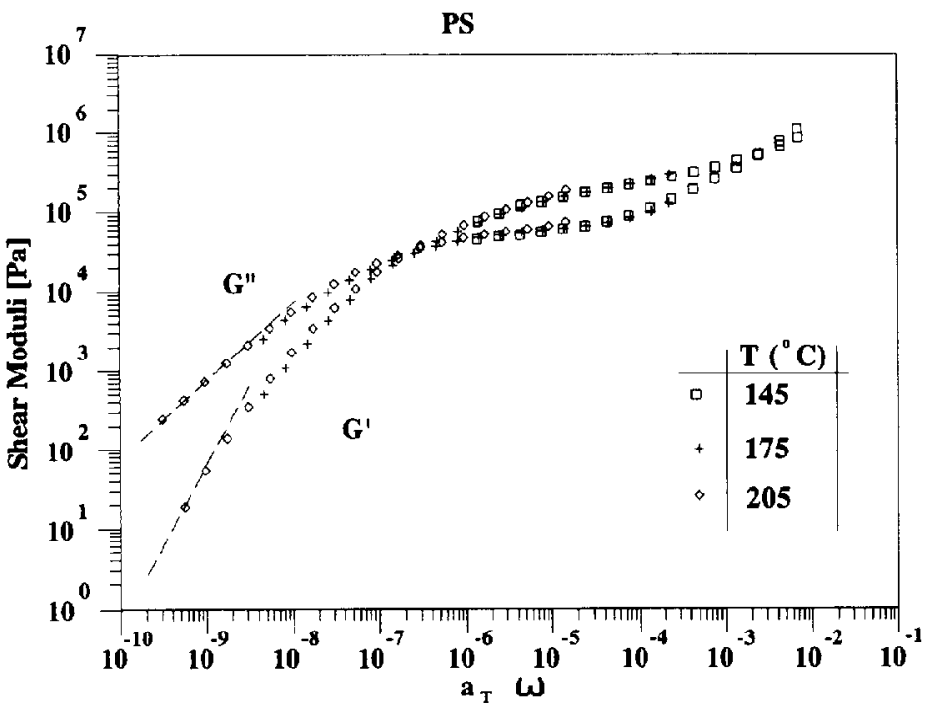

FIG. 4. Time-temperature superposition was applied to the complex moduli of polystyrene, measured at temperatures between 145 and $175^{\circ} \mathrm{C}$. The shift factors were correlated with the WLF equation, and extrapolated to present the data with $T_{\text {ref }}=110^{\circ} \mathrm{C}$. The dotted lines have slopes of 1 and 2 .

Fig. 3. Glass transition temperatures are practically independent of molecular weight and tacticity for high molecular weight PS/PVME blend systems and their values were adopted from the measurements of Yang et al. (1986).

\section{Isothermal dynamic mechanical measurements}

Dynamic mechanical measurements selectively probe relaxation modes with time constants around $\lambda \approx 2 \pi / \omega$, where $\omega$ is the frequency. The experimental frequency range was 0.01 to $100 \mathrm{rad} / \mathrm{s}$. The frequency-dependent complex modulus of the pure components was measured at a range of temperatures and shifted onto master curves. The horizontal shift factor followed the WLF equation

$$
\log a_{T}=-c_{1}\left(T-T_{\text {ref }}\right) /\left[c_{2}+\left(T-T_{\text {ref }}\right)\right]
$$

Data for polystyrene at 145,175 , and $205^{\circ} \mathrm{C}$ gave WLF coefficients of $c_{1}=12.5$ and $c_{2}=63.7 \mathrm{~K}$ ( $T_{\text {ref }}$ was $110^{\circ} \mathrm{C}$ as discussed later $)$, while 


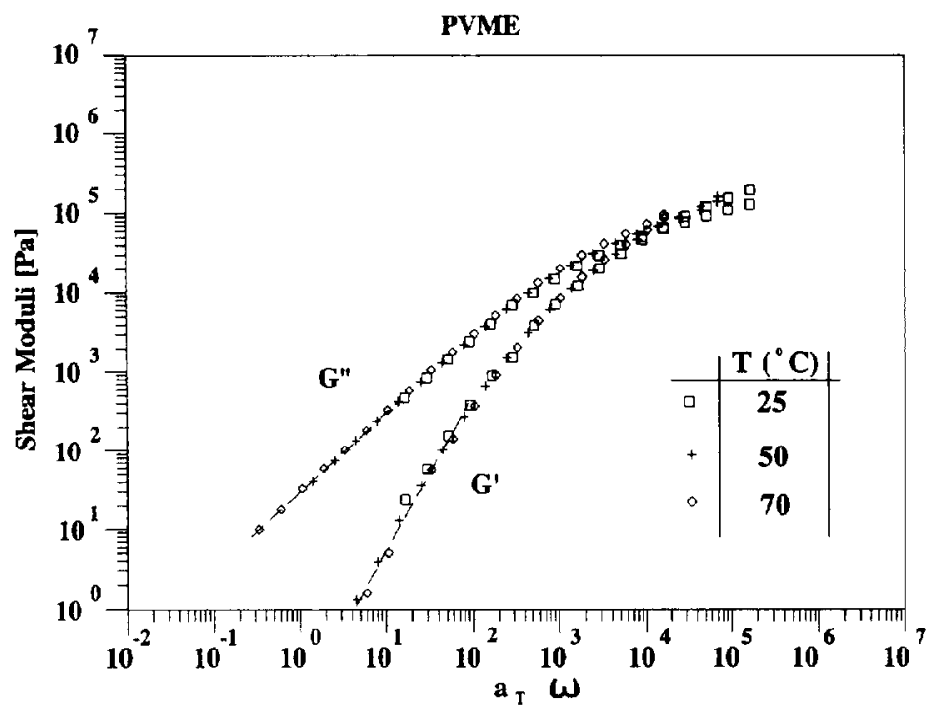

FIG. 5. Time-temperature superposition was applied to the complex moduli of poly(vinyl methyl ether) measured at temperatures between 25 and $70^{\circ} \mathrm{C}$. The shift factors were correlated with the WLF equation, and extrapolated to present the data with $T_{\text {ref }}=110^{\circ} \mathrm{C}$. The dotted lines have slopes of 1 and 2 .

PVME data were measured at 25,51 , and $71^{\circ} \mathrm{C}$ and fit with $c_{1}=323.5$ and $c_{2}=8597.1 \mathrm{~K}$, also with $T_{\text {ref }}=110^{\circ} \mathrm{C}$ (close to Arrhenius behavior). The vertical shift factor was very small $\left(\log b_{T}<0.1\right)$.

The dynamic mechanical data for the two pure components are shown in Figs. 4 and 5. The corresponding spectra are listed in Tables I and II. For later comparison with the blend behavior, we are interested in the moduli at temperatures around $110^{\circ} \mathrm{C}$ which is close to the LCST. However, measurements at $110^{\circ} \mathrm{C}$ were not possible in PS due to the proximity of $T_{g}$ and in PVME due to the extremely low torque levels in the rheometer. Instead, the shift factors were correlated with Eq. (1) and extrapolated to $110^{\circ} \mathrm{C}$. With a reference temperature of $110^{\circ} \mathrm{C}$, we observe that the terminal relaxation time of PS is about 10 orders of magnitude higher than that of PVME.

The samples had to be stable during rheological measurements over all frequencies (otherwise the effects of time and frequency would be coupled). This was ensured by time sweeps at a constant frequency; the 
TABLE I. The discrete relaxation spectrum for $P S$ at $146^{\circ} \mathrm{C}$. The relaxation modulus is given as a sum of Maxwell modes: $G(t)=\Sigma g e^{-t / \lambda_{i}}$.

\begin{tabular}{cl}
\hline \hline$g_{i}\left(\mathrm{~Pa}_{\mathrm{a}}\right.$ & \multicolumn{1}{c}{$\lambda_{i}(\mathrm{~s})$} \\
\hline $0.4102 E 7$ & $0.5765 E-3$ \\
$0.2405 E 6$ & $0.7592 E-2$ \\
$0.1109 E 6$ & $0.3313 E-1$ \\
$0.8573 E 5$ & $0.1844 E 0$ \\
$0.6225 E 5$ & $0.1291 E 1$ \\
$0.5738 E 5$ & $0.6827 E 1$ \\
$0.4307 E 5$ & $0.4404 E 2$ \\
$0.1985 E 5$ & $0.2453 E 3$ \\
$0.5895 E 4$ & $0.1053 E 4$ \\
$0.8579 E 3$ & $0.5307 E 4$ \\
$0.1526 E 2$ & $0.1884 E 6$ \\
\hline \hline
\end{tabular}

$G^{\prime}$ and $G^{\prime \prime}$ for samples near the LCST reached a quasi-steady state after approximately $100 \mathrm{~min}$ as shown in Fig. 6. Below the LCST, the moduli increased with time and quickly reached steady state, but for samples heated rapidly to temperatures above the LCST, the moduli decreased with time and, after approximately $100 \mathrm{~min}$, they reached a quasi-steady state, where the changes were very slow $(0.2 \% / \mathrm{min})$.

After the prescribed annealing above the LCST, the samples were rapidly quenched below their $T_{g}$ by liquid nitrogen and the morphology was studied by transmission electron microscopy (Fig. 7). In the PS/ PVME system the contrast occurs due to a preferential degradation of the PVME (more details are available in the studies of Voigt-Martin $e t$ al. 1986). The size of the phase separated domains was in the range of 0.1 to $2 \mu$. The phases exhibited considerable interpenetration and connectivity.

TABLE II. The discrete relaxation spectrum for PVME at $70^{\circ} \mathrm{C}$.

\begin{tabular}{cl}
\hline \hline$g_{i}(\mathrm{~Pa})$ & \multicolumn{1}{c}{$\lambda_{i}(\mathrm{~s})$} \\
\hline $0.4800 E 6$ & $0.7178 E-4$ \\
$0.1595 E 6$ & $0.5402 E-3$ \\
$0.7200 E 5$ & $0.3524 E-2$ \\
$0.1769 E 5$ & $0.2064 E-1$ \\
$0.1822 E 4$ & $0.1200 E 00$ \\
$0.9340 E 1$ & $0.2321 E 01$ \\
\hline \hline
\end{tabular}




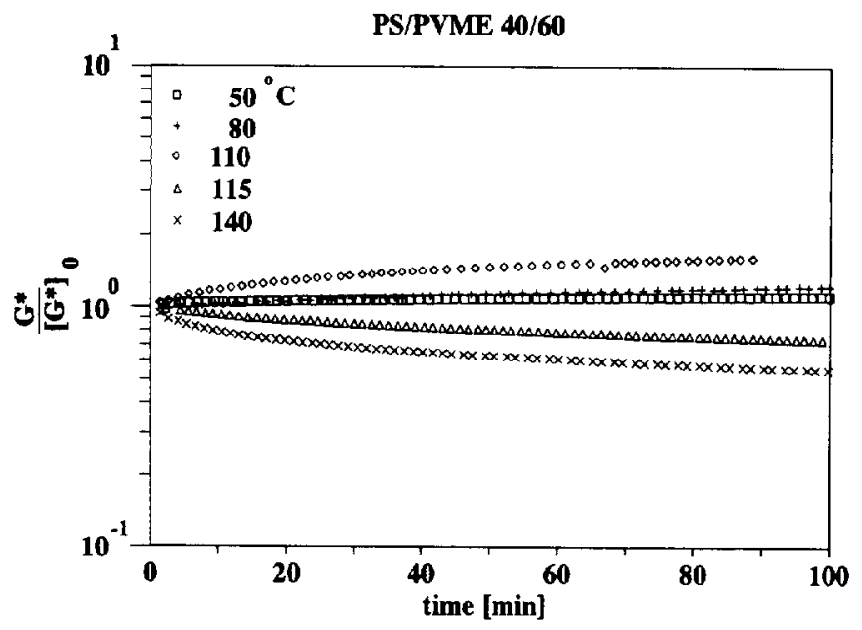

FIG. 6. The complex modulus at a constant frequency $(0.1 \mathrm{rad} / \mathrm{s})$ is examined as a function of time at different temperatures for a 40/60 PS/PVME blend.

Strain sweeps at constant frequency showed linear viscoelastic behavior up to strain amplitudes of approximately 0.2. In the frequency sweeps, the samples were subjected to a strain of 0.2 at the lowest frequency $(0.01 \mathrm{rad} / \mathrm{s})$ in order to obtain sufficient torque signal. At higher frequencies the imposed strains were progressively lowered.

In the single-phase region, the complex modulus was obtained over eight decades of frequency using time-temperature superposition (Fig. 8 and Table III for the 40/60 PS/PVME blend). For the $40 / 60$ blend at low frequencies, $G^{\prime \prime}(\omega)$ was greater than $G^{\prime}(\omega)$ and the slopes were 1.0 and $1.7( \pm 0.1)$, respectively, on a $\log -\log$ scale, indicating that the sample was close to its terminal behavior. At higher frequencies $G^{\prime}$ exceeded $G^{\prime \prime}$ and the change in the shear moduli with frequency was relatively small (plateau region of $G^{\prime}$ ); this was followed by a second crossover of $G^{\prime}$ and $G^{\prime \prime}$ and a transition to glassy behavior. Similar plots have been obtained for the 20/80 PS/PVME and the 60/40 PS/PVME and we will describe these in conjunction with the phase transition data. The shift factors $a_{T}$ are plotted against the reciprocal of temperature in Fig. 9. The solid line is the WLF fit to the data with $C_{1}=4.4$ and $C_{2}=182.8$, the WLF constants of the 40/60 PS/PVME blend were reduced to $T_{g}\left(-25^{\circ} \mathrm{C}\right)$, the constants were $C_{1 g}=15.1$, and $C_{2 g}=53.4$ 


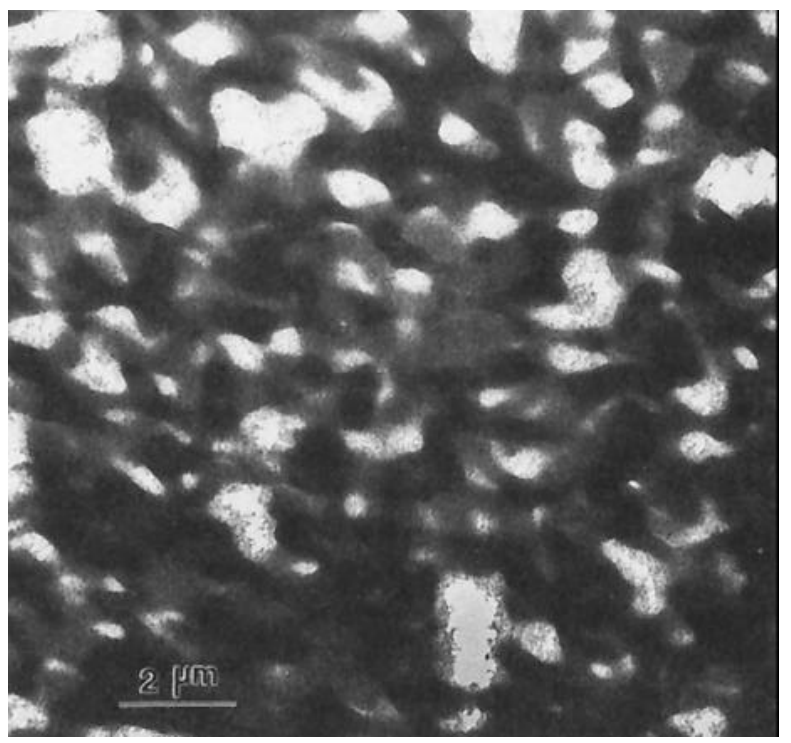

FIG. 7. A $40 / 60 \mathrm{PS} / \mathrm{PVME}$ sample was thermally equilibrated at $120^{\circ} \mathrm{C}$ for $100 \mathrm{~min}$; the sample was then quenched under liquid nitrogen and approximately $0.1 \mu \mathrm{m}$ sections were cryomicrotomed at $-100^{\circ} \mathrm{C}$. The sections were imaged by TEM with an accelerating voltage of $100 \mathrm{kV}$.

$\mathbf{K}$, which are in good agreement with values calculated from temperature-dependent fluorescence decay measurements by Halary $e t$ al. (1988).

At temperatures above the LCST, the dynamic moduli of a $20 / 80$ PS/PVME sample were shifted along the frequency axis assuming that the high frequency behavior was not affected by the phase transition, Fig. 10. At low frequencies, the phase transition was accompanied by a large increase in the shear storage and loss moduli. $G^{\prime}$ and $G^{\prime \prime}$ were proportional to $\omega^{0.74 \pm 0.01}$ over three decades of frequency. We could not extend these measurements to higher temperatures due to transducer limitations. The phase transition in the 40/60 PS/PVME blend was also accompanied with a large increase in the shear moduli at low frequencies; $G^{\prime}$ and $G^{\prime \prime}$ were proportional to $\omega^{0.85 \pm 0.02}$, see Fig. 11 . For the $60 / 40$ blend, the complex moduli did not exhibit any measurable changes near the phase transition temperature, within our window of 


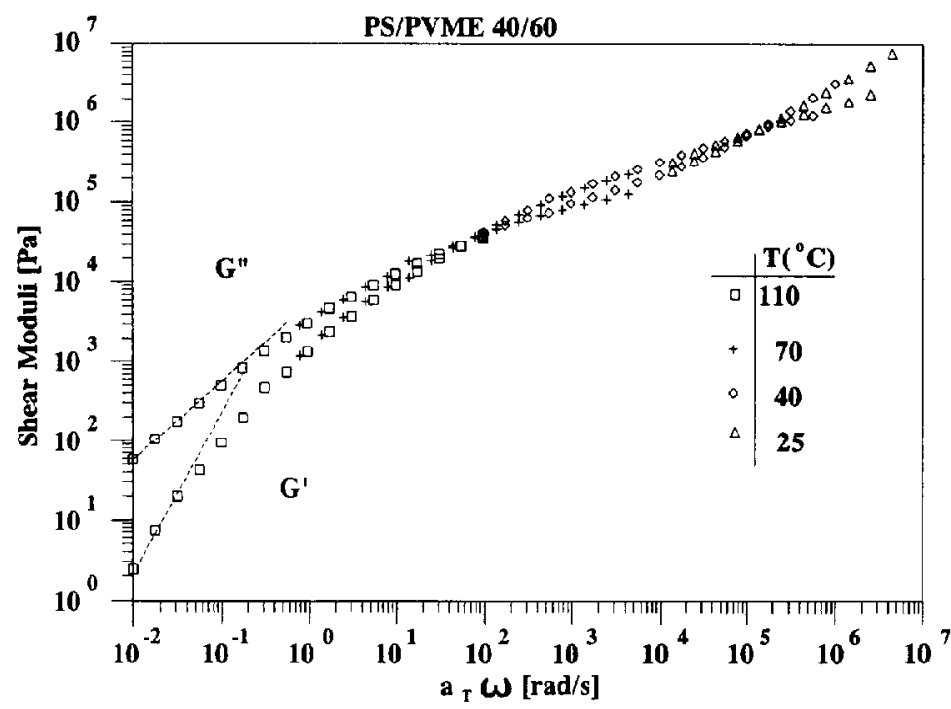

FIG. 8. Complex moduli of a $40 / 60$ PS/PVME blend in the single-phase region. Data at several temperatures have been shifted in frequency to yield a master curve with $110^{\circ} \mathrm{C}$ as the reference. The dotted lines have slopes of 1 and 2.

observation. Heating to higher temperatures was required to significantly increase $G^{\prime}$ and $G^{\prime \prime}$ at low frequencies as shown in Fig. 12. Degradation at temperatures above $155^{\circ} \mathrm{C}$ precluded measurements of the terminal zone for these samples.

TABLE 1II. The discrete relaxation spectrum for a $40 / 60 \mathrm{PS} / \mathrm{PVME}$ blend at $110^{\circ} \mathrm{C}$.

\begin{tabular}{cl}
\hline \hline$g_{i}(\mathrm{~Pa})$ & \multicolumn{1}{c}{$\lambda_{i}(\mathrm{~s})$} \\
\hline $0.4253 E 7$ & $0.6958 E-6$ \\
$0.3796 E 6$ & $0.1984 E-4$ \\
$0.2016 E 6$ & $0.2400 E-3$ \\
$0.8668 E 5$ & $0.2513 E-2$ \\
$0.4086 E 5$ & $0.1641 E-1$ \\
$0.1324 E 5$ & 0.1090 \\
$0.3744 E 4$ & 0.5925 \\
$0.9212 E 3$ & $0.3565 E 1$ \\
$0.4183 E 2$ & $0.3726 E 2$ \\
\hline \hline
\end{tabular}




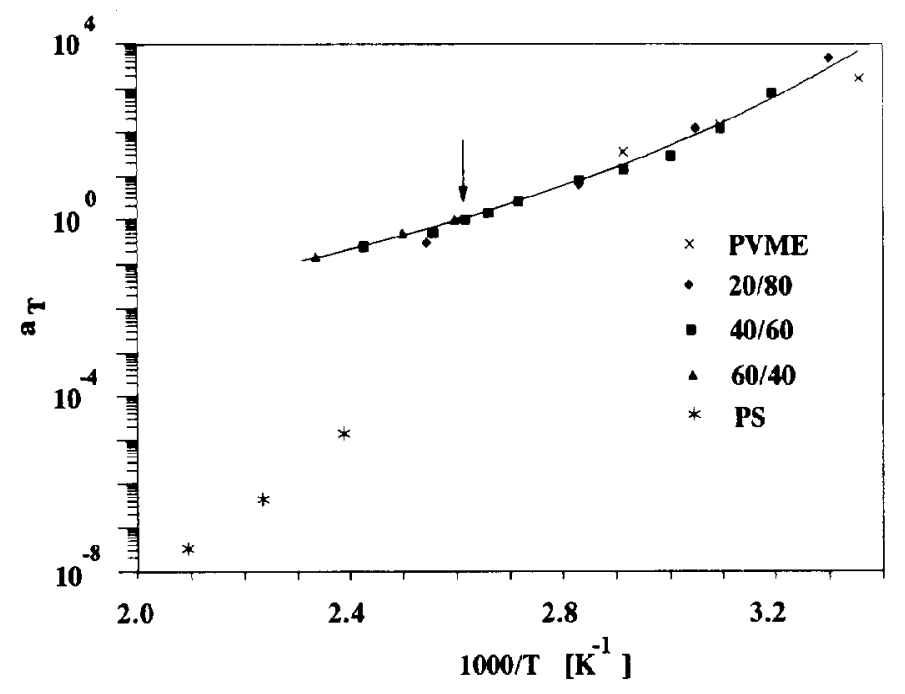

FIG. 9. The shift factors for a 40/60 PS/PVME sample plotted against the reciprocal of absolute temperature. The solid line is the WLF fit to the data with $C_{1}=4.4$ and $C_{2}$ $=182.8 \mathrm{~K}$. The arrow is at $110^{\circ} \mathrm{C}$, the phase separation temperature for the $40 / 60$ PS/PVME blend. Shift factors for the 20/80 PS/PVME, 60/40 PS/PVME, PS, and PVME are also included for comparison.

\section{Measurements under slow heating}

Phase separation had the largest observable effect on the complex modulus of blends with low PS content and at low frequencies. This can be seen for the 40/60 PS/PVME sample which had been equilibrated at $100^{\circ} \mathrm{C}$ for $2 \mathrm{~h}$. The sample was heated at $0.5 \mathrm{~K} / \mathrm{min}$ (Fig. 13) while measuring $G^{\prime}$ and $G^{\prime \prime}$ at $0.1 \mathrm{rad} / \mathrm{s}$ along with the fluorescence intensity. In the single-phase region, the complex moduli decreased steadily with increasing temperature until the sample reached the phase transition temperature (as detected by in situ fluorescence measurements). Then, $G^{\prime}$ increased rapidly by almost an order of magnitude and $G^{\prime \prime}$ increased by approximately $200 \%$; at higher temperatures they decreased with temperature again. It is important to perform these experiments at low frequency. At a higher frequency of $10 \mathrm{rad} / \mathrm{s}$ (Fig. 14), the phase transition temperature has no noticeable effect on the complex moduli $G^{\prime}$ and $G^{\prime \prime}$. 


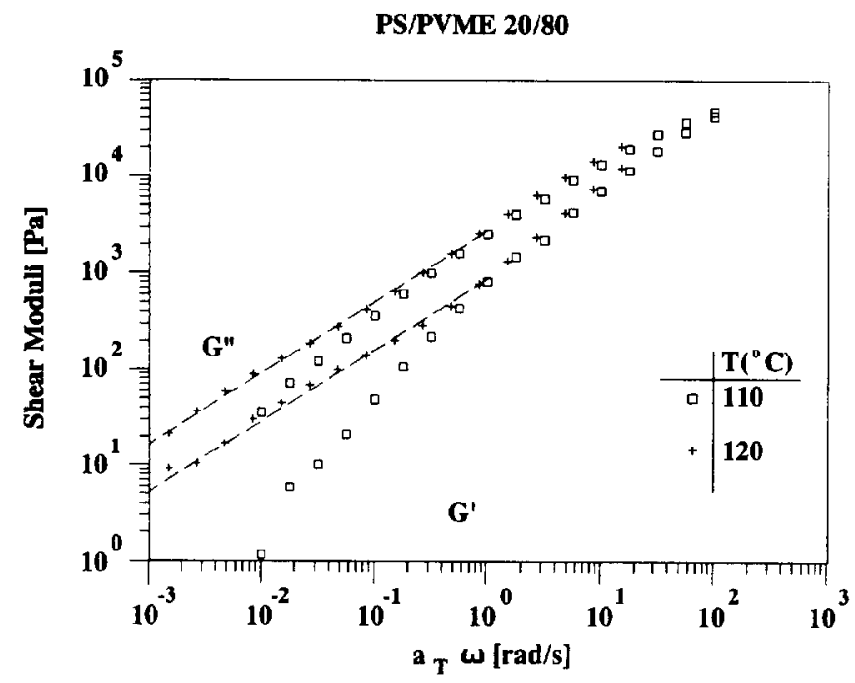

FIG. 10. Moduli measurements in isothermal frequency sweeps for 20/80 PS/PVME samples near the LCST. Time-temperature superposition was performed with $T_{\text {ref }}$ $=110^{\circ} \mathrm{C}$ assuming that the phase transition does not affect the high frequency behavior. The lines in the low frequency region have a slope of 0.75 .

\section{DISCUSSION}

In this section, some additional comments are made on the $G^{\prime}, G^{\prime \prime}$ data measured in the single-phase region, and some of the efforts to model the rheological behavior of blends in the single-phase region are briefly described. This is followed by a more extensive discussion of the data measured in the phase-separated region.

In the single phase region, the dynamic moduli of PS/PVME blends can be shifted into master curves by time-temperature superposition. The horizontal shift factors $a_{T}$ of the blends are very similar to the shift factors of the pure PVME as shown in Fig. 8, suggesting that the mobility of the PVME molecules is mostly responsible for the rheological behavior of the blend. The solid line in Fig. 9 represents the WLF fit through the blend data suggesting that composition has very little influence in the range $20 \%$ to $60 \%$ PS. The shift factors for pure PS at $100^{\circ} \mathrm{C}$ are extremely sensitive to temperature because of the proximity to its glassy state; this is manifested in an increase in the zero-shear 


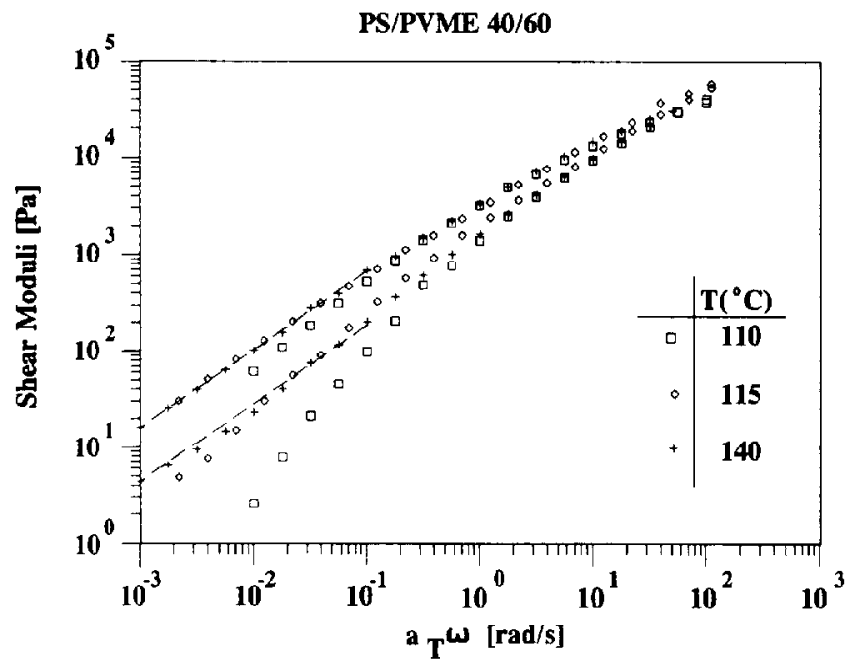

FIG. 11. Time-temperature superposition was applied to the moduli of $40 / 60 \mathrm{PS} / \mathrm{PVME}$ samples, with the $T_{\text {ref }}=110^{\circ} \mathrm{C}$. The lines in the low frequency region have a slope of 0.85 .

viscosity with increasing PS content in the single-phase region, and a shift in the terminal behavior to longer times, as shown by comparing Figs. 15, 16, and 17.

There is considerable interest in predicting the rheological behavior of blends in the single phase region from the pure component rheology but the studies are not yet conclusive. For example, Watanabe and Kotaka (1991) examined the viscoelastic properties of blends of nearly monodisperse polystyrene with molecular weights $M_{L}$ and $M_{S}$ (long and short chains). In a dilute blend where the weight fraction of long chains was small, the longer chains seemed to entangle only with the matrix chains and not amongst themselves. When $M_{L}>M_{S}$, the relaxation of the long chains could be described by a "constrained-release" (CR) model formulated on the basis of Rouse dynamics. When $M_{S} \rightarrow M_{L}$, a configuration-dependent CR model was developed where a competition between reptation and Rouse dynamics was considered; this was especially successful in describing the terminal relaxation.

There are also several interesting modeling studies of the rheological response of binary blends with same chemical composition but different molecular weights; e.g., Onogi et al. (1970) and Schausberger (1986) 


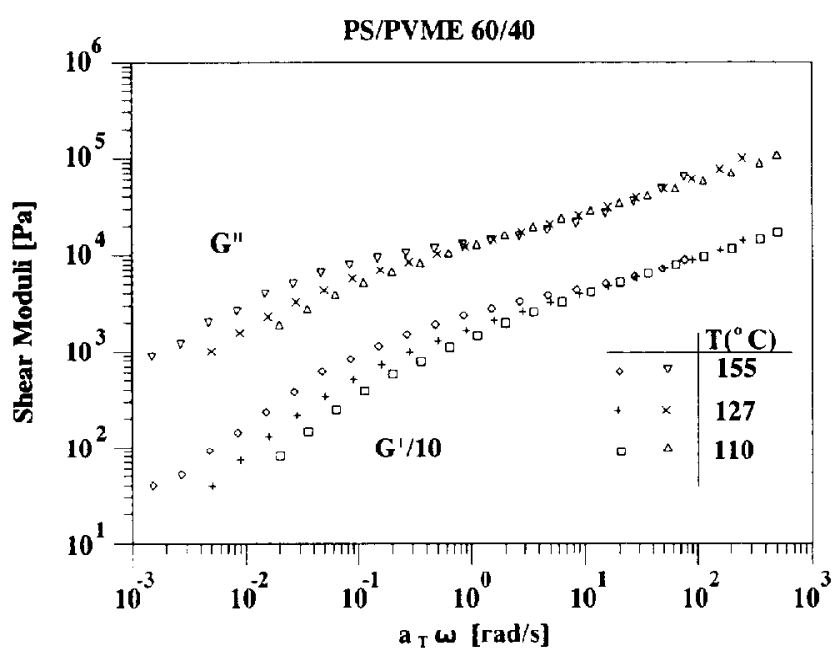

FIG. 12. Time-temperature superposition was applied to the moduli of 60/40 PS/PVME samples, with $T_{\text {ref }}=110^{\circ} \mathrm{C}$. For clarity the $G^{\prime}$ values have been divided by 10 .

for blends of polystyrenes, Struglinski and Graessley (1986) in blends of polybutadienes, and Utracki (1989) in blends of linear low density polyethylene with low density polyethylene. However, the PS/PVME blend is a more complicated system, partly because the glass transition temperature is so much lower for PVME than for PS. The region between terminal relaxation and glassy behavior in the $G^{\prime}, G^{\prime \prime}$ data occurs over different frequency windows for PS, PVME, and the blend, as can be seen by comparing Figs. 4, 5, and 6 (or from the corresponding relaxation spectra in Tables I, II, and III). PS and PVME have different chemical structures, both have broad molecular weight distributions, and the glass transition temperature of the blends has a strong and nonlinear composition dependence. More work is needed in this direction.

Figures 10-12 show the influence of phase separation on $G^{\prime}$ and $G^{\prime \prime}$ of the three samples. The 20/80 and 40/60 PS/PVME blends were observed near the terminal region; phase separation resulted in a large increase in the shear moduli, and $G^{\prime}$ and $G^{\prime \prime}$ followed a power law with frequency. A similar evolution of $G^{\prime}$ and $G^{\prime \prime}$ from terminal liquid behavior (slopes of 2 and 1 with frequency) to a power law with frequency (slope of 0.5 ) was observed when diblock copolymers of polybutadiene 


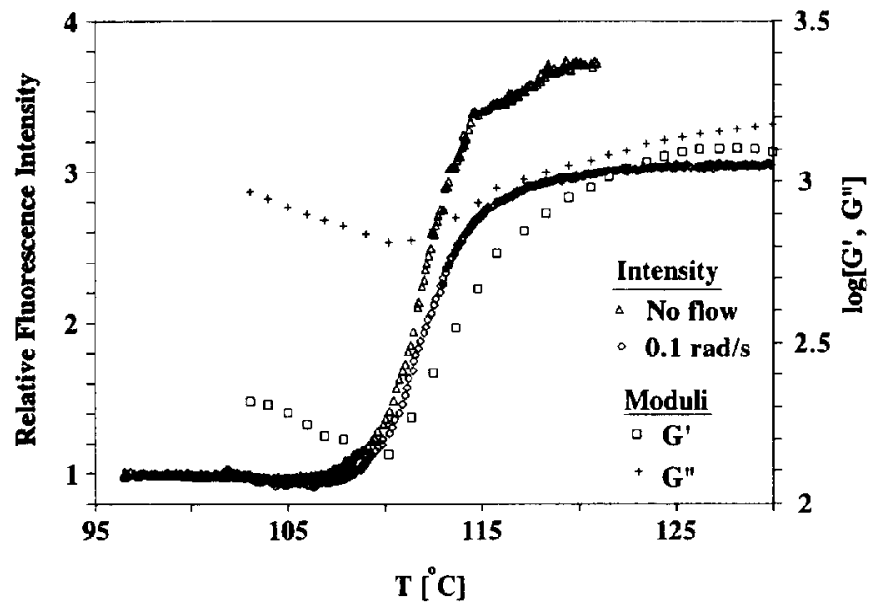

FIG. 13. After equilibration at $100^{\circ} \mathrm{C}$, a $40 / 60 \mathrm{PS} / \mathrm{PVME}$ sample was heated at 0.5 $\mathrm{K} / \mathrm{min}$; the $G^{\prime}$ and $G^{\prime \prime}$ were measured at $0.1 \mathrm{rad} / \mathrm{s}$, and in situ fluorescence measurements detected the onset of phase separation.

underwent a microphase separation transition (Bates, 1984). We were unable to determine if the power law behavior in the $G^{\prime}, G^{\prime \prime}$ of the phase-separated blend was restricted to certain intermediate frequencies (with liquid-like response at much lower frequencies) or if the power law relaxation behavior extended to zero frequency as it does in polymers near the gel point (Chambon and Winter, 1985).

The power law relaxation behavior persisted to temperatures well above the LCST. This could be interpreted in several ways: (1) the power law behavior is associated with the critical slowing down of the relaxations which determined by the proximity to the LCST or (2) the power law relaxation is determined by the phase-separated morphology. The second hypothesis is in agreement with the experiments of Stadler et al. (1980).

For the 20/80 and the 40/60 PS/PVME blends, the effect of phase separation on $G^{\prime}$ was much larger than on $G^{\prime \prime}$ in agreement with the scaling analysis of Ajji and Choplin (1991), although their analysis was performed for near-critical mixtures. In this study $G^{\prime \prime}$ also exhibited an increase with phase separation; we found that both the Cole-Cole rep- 


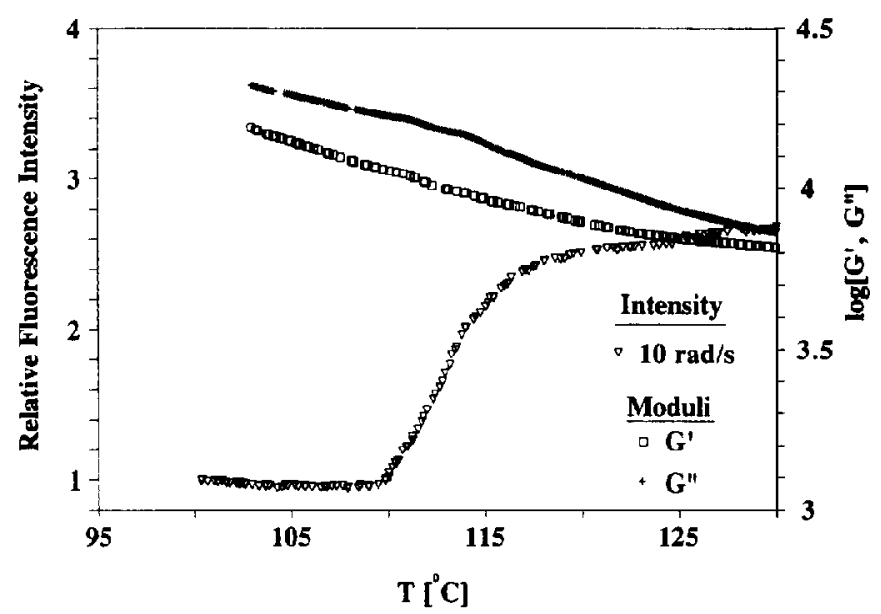

FIG. 14. Using the same procedure described in Fig. 12, the $G^{\prime}$ and $G^{\prime \prime}$ were measured at $10 \mathrm{rad} / \mathrm{s}$.

resentation and the frequency dependence of the $G^{\prime}$ and $G^{\prime \prime}$ were equally sensitive to phase separation in contrast to the results of Ajji et al. (1989).

However, the complex moduli of the 60/40 PS/PVME blend did not exhibit any measurable changes near the phase transition temperature; this is consistent with the results obtained by Stadler et al. (1988). Near the quiescent phase transition temperature, $110^{\circ} \mathrm{C}$, the $G^{\prime}$ and $G^{\prime \prime}$ of the 60/40 PS/PVME sample were measured near the plateau region. We could not access the terminal zone by measuring at temperatures much higher than $155^{\circ} \mathrm{C}$ because of degradation. This degradation problem might have been the reason why Stadler et al. (1988) did not thermally equilibrate their samples (which would have required approximately $100 \mathrm{~min}$ ) before measuring the complex modulus, which complicates interpretation of their data.

Phase separation was accompanied with much larger increases in the complex modulus at low frequencies than the shear stresses and first normal stress differences measured during steady shear experiments by Mani et al. (1991). This is probably because dynamic mechanical measurements probe specific relaxation modes of the material depending on 


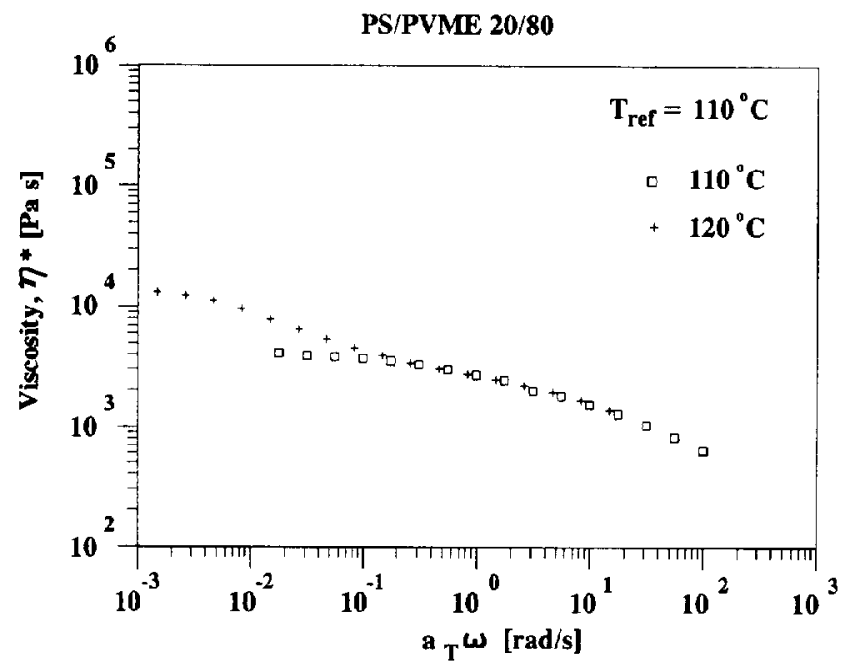

FIG. 15. Effect of phase separation on the complex viscosity of a 20/80 PS/PVME sample. The single-phase data were measured at $110^{\circ} \mathrm{C}$; data for the phase-separated blend were measured at $120^{\circ} \mathrm{C}$ and shifted to $110^{\circ} \mathrm{C}$

the frequency of measurement, whereas in steady state measurement the response is summed over all modes and an interpretation becomes model dependent.

The influence of phase separation on the dynamic mechanical response is expected to be twofold: the interface (or region of steep composition gradient between the separating components) imposes a constraint for the molecular motion. Secondly, the bulk properties of the phases with high PS content are many orders of magnitude different from those of the phases with low PS content; this second effect is expected to dominate the mechanical behavior. We proceed to examine the three samples in more detail for qualitative understanding. When a 20/80 PS/PVME sample (glass transition temperature at approximately $-25^{\circ} \mathrm{C}$ ) is heated to a temperature of $\Delta T=10 \mathrm{~K}$ above the coexistence temperature, the composition of the phases can be estimated from the phase diagram in Fig. 3. The flat shape of the phase diagram results in an extreme composition difference between the two phases, a PS-rich phase (approximately $80 \%$ PS) with a glass transition temperature of approximately $50^{\circ} \mathrm{C}$ and a PVME-rich phase (approximately 


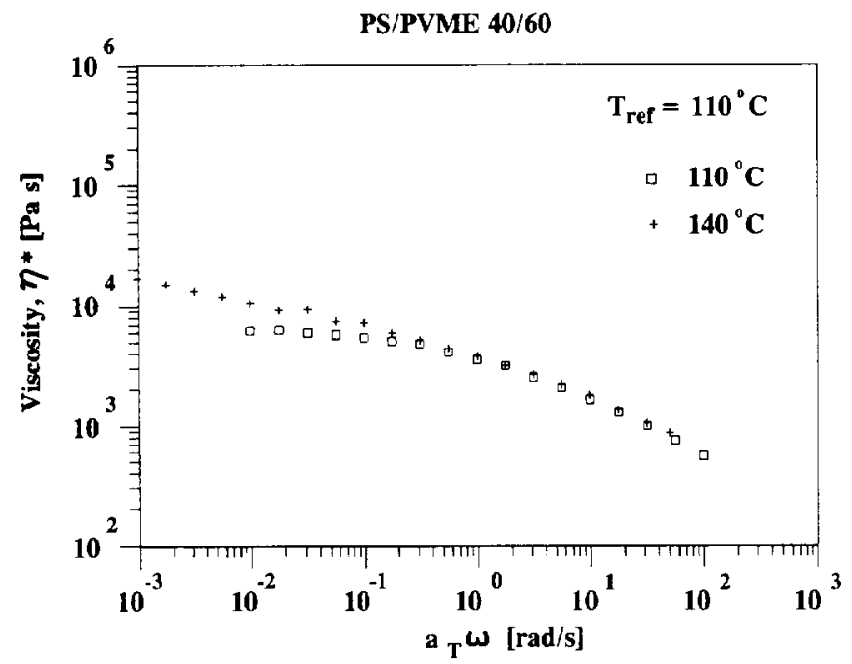

FIG. 16. Effect of phase separation on the complex viscosity of a $40 / 60 \mathrm{PS} / \mathrm{PYME}$ sample. The single-phase data were measured at $110^{\circ} \mathrm{C}$; the data for the phase-separated blend were measured at $140^{\circ} \mathrm{C}$ and shifted to $110^{\circ} \mathrm{C}$.

15\% PVME) with a glass transition temperature of approximately $-25^{\circ} \mathrm{C}$. Although the volume fraction of the PS-rich phase will be small (dictated by the lever rule) its effect on the overall behavior will be dominating since the bulk properties of the PS-rich fraction exceeds that of the PVME-rich fraction by several orders of magnitude. For the 40/60 PS/PVME blend, the behavior is qualitatively similar; the change in $T_{g}$ is not as large and consequently the increase in the complex moduli is not as large as for the $20 / 80$ sample. A more extreme case is the 60/40 PS/PVME sample, which required a much larger $\Delta T$ to change the complex moduli. The above arguments indicate that influence of phase separation on the rheology depends on the distance from the glass transition temperature, which in turn depends on the composition of the specific blend sample.

Phase separation also has a large effect on the complex viscosity, especially at low frequencies as shown by replotting the data from Figs. 10-12 in Figs. 15-17. It is interesting that the viscosity accompanying phase separation in critical fluids was predicted to be enhanced by a factor of 2 by Onuki (1987). This prediction was strictly based on the 


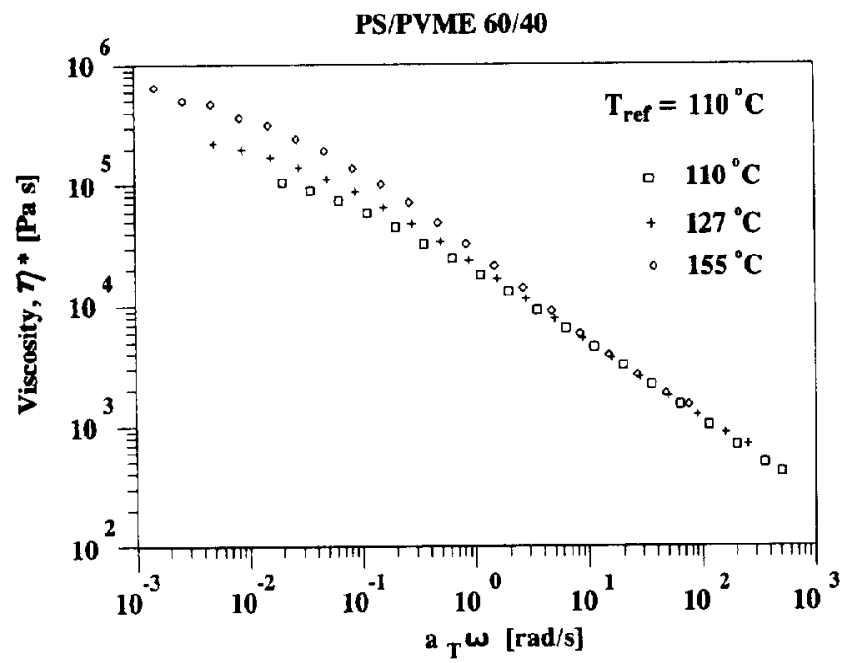

FIG. 17. Effect of phase separation on the complex viscosity of a 60/40 PS/PVME sample. The single-phase data were measured at $110^{\circ} \mathrm{C}$; data for the phase-separated blend were measured at 127 and $155^{\circ} \mathrm{C}$ and shifted to $110^{\circ} \mathrm{C}$.

appearance of the interface. It did not account for differences in the bulk properties of the two phases which may be very significant as shown by this study.

\section{CONCLUSIONS}

Phase separation in the PS/PVME blend results in a significant increase in the low-frequency complex moduli, while the high frequency behavior is not affected. The sensitivity of low-frequency complex moduli to the onset of phase separation is confirmed by experiments done under slow heating along with in situ fluorescence measurements. The large effect on the moduli is attributed to the enormous differences between the moduli of the PS-rich and the PVME-rich phases in the phase-separated blend. At low frequencies, $G^{\prime}(\omega)$ and $G^{\prime \prime}(\omega)$ of the phase-separated blends followed a power law with frequency, even at temperatures much above the LCST. The terminal behavior at low frequencies is not accessible.

In the single-phase region, the PS/PVME blends behaved like typical viscoelastic liquids of broad molecular weight distribution. $G^{\prime}$ and $G^{\prime \prime}$ 
exhibited a broad transition from the entanglement regime to the terminal zone, with slopes of 1.7 and 1.0 at the lowest accessible frequencies. With increasing PS content, the terminal region shifted to even longer times and the zero-shear viscosity increased.

\section{ACKNOWLEDGMENTS}

We are grateful to J. L. Halary and L. Monnerie for supplying the labeled polystyrene. The molecular weight distribution of the polystyrene and poly (vinyl methyl ether) was determined using gel permeation chromatography by J. Swadesh at Polymer Labs, Amherst, MA. This project was funded by Center for University of Massachusetts Industrial Research on Polymers, CUMIRP, and by the donors of the Petroleum Research Fund, administered by the American Chemical Society (ACSPRF\#23207-AC7).

\section{References}

Ajii, A., L. Choplin, and R. E. Prud'homme, "Rheology and phase separation in polystyrene/poly(vinyl methyl ether) blends," J. Polym. Sci. B: Polym. Phys. 26, 2279-2289 (1988).

Ajji, A. and L. Choplin, "Rheology and dynamics near phase separation in a polymer blend: model and scaling analysis," Macromolecules 24, 5221-5223 (1991).

Araujo, M. A. and R. Stadler, "Viscoelastic properties of mixtures of polystyrene with low-molecular weight poly[oxy(2,6-dimethyl-1,4-phenylene)]," Makromol. Chem. 189, 2169-2186 (1988).

Bates, F. S., "Block copolymers near the microphase separation transition. 2. Linear dynamic mechanical properties," Macromolecules 17, 2607-2613 (1984).

Baumgaertel, M. and $\mathbf{H}$. H. Winter, "Determination of the discrete relaxation and retardation time spectra from dynamic mechanical data," Rheol. Acta 28, 511-519 (1989).

Bird, R. B., R. Armstrong, and O. Hassager, Dynamics of Polymeric Liquids, 3rd ed. (Wiley, New York, 1977), p. 303

Brekner, M. J., H. J. Cantow, and H. A. Schneider, "Interactions in compatible polymer systems. 1. Viscoelasticity and glass transition of polystyrene/poly(vinyl methyl ether) blends," Polym. Bull. 14, 17-24 (1985).

Cavaille, J. Y., J. Perez, C. Jourdan, and G. P. Johari, "Dynamic mechanical behavior of poly (vinylmethyl ether)/polystyrene blends," J. Polym. Sci. B: Polym. Phys. 25, 1847-1858 (1987)

Chambon, F. and H. H. Winter, "Stopping of crosslinking reaction in a PDMS polymer at the gel point," Polym. Bull. 13, $499-503$ (1985)

Ferry, J. D., Viscoelastic Properties of Polymers, 3rd ed. (Wiley, New York, 1960).

Fischer, E. W., G. P. Hellmann, H. W. Spiess, F. J. Hörth, U. Ecasius, and M. Wehrle, "Mechanical properties, molecular motions and density fluctuations in polymeradditive mixtures," Makromol. Chem. Suppl. 12, 189-214 (1985). 
Frederickson, G. H. and R. G. Larson, "Viscoelasticity of homogeneous polymer melts near a critical point," J. Chem. Phys. 86, 1553-1560 (1987).

Halary, J. L. and L. Monnerie, "Fluorescence quenching of anthracene-labelled polystyrene by poly (vinyl methyl ether): a new approach for the analysis of phase separation phenomena," in Photophysical and Photochemical Tools in Polymer Science, edited by M. A. Winnik (Reidel, Dordrecht, The Netherlands, 1986), pp. 589-610.

Halary, J. L., J. M. Ubrich, L. Monnerie, H. Yang, and R. S. Stein, "Isotope effects on the phase separation in polystyrene-poly (vinyl methyl ether) blends," Polym. Commun. 26, 73-76 (1985).

Halary, J. L., J. M. Ubrich, J. M. Nunzi, L. Monnerie, and R. S. Stein, "Phase separation in polystyrene-poly (vinyl methyl ether) blends: a fluorescence emission analysis," Polymer 25, 956-962 (1984)

Halary, J. L., F. B. C. Larbi, P. Oudin, and L. Monnerie, "Molecular mobility in polystyrene-poly (methyl vinyl ether) blends as studied by fluorescence polarization," Makromol. Chem. 189, 2117-2124 (1988).

Katsaros, J. D., M. F. Malone, and H. H. Winter, "The effects of flow on miscibility in a blend of polystyrene and poly( vinyl methyl ether)," Poly. Eng. Sci. 29, 1434-1445 (1989).

Landry, C. J. T. and P. M. Henrichs, "The influence of blending on the local motions of polymers: studies involving polycarbonate, poly (methyl methacrylate), and a polyester," Macromolecules 22, 2157-2166 (1989).

Mani, S., M. F. Malone, H. H. Winter, J. L. Halary, and L. Monnerie, "Effects of shear on miscible polymer blends: In situ fluorescence studies," Macromolecules 24, 54515458 (1991).

Mazich, K. A. and S. H. Carr, "The effect of flow on the miscibility of a polymer blend." J. Appl. Phys. 54, 5511-5514 (1983).

Onogi, S., T. Masuda, and K. Kitagawa, "Rheological properties of anionic polystyrenes. I. Dynamic viscoelasticity of narrow-distributed polystyrenes," Macromolecules 3, 109-125 (1970).

Onuki, A., "Viscosity enhancement by domains in phase-separating fluids near the critical point: Proposal of critical rheology," Phys. Rev. A 35, 5149-5155 (1987).

Onuki, A., "Spinodal decomposition and nucleation in the presence of flow," Int. J. Thermophys. 2, 293-308 (1989).

Paul, D. R., in Polymer Blends, edited by D. R. Paul and S. Newman (Academic, New York, 1978), Vol. 1.

Prest, W. M. and R. S. Porter, "Rheological properties of poly(2,6-dimethylphenylene oxide )-polystyrene blends," J. Poly. Sci. A-2 10, 1639-1655 (1972).

Schausberger, A., "A simple method of evaluating the complex moduli of polystyrene blends," Rheol. Acta 25, 596-605 (1986).

Schneider, H. A. and M. J. Brekner, "Thermodynamic aspects of the glass-transition in the compatible blend polystyrene-poly (vinyl methyl ether)," Polym. Bull. 14, 173-178 (1985),

Stadler, R., L. L. Freitas, V. Krieger, and S. Klotz, "Influence of phase separation on the linear viscoelastic properties of a polystyrene-poly(vinyl methyl ether) blend," Polymer 29, 1643-1648 (1988).

Struglinski, M. J. and W. W. Graessley, "Effects of polydispersity on the linear viscoelastic properties of entangled polymers. I. Experimental observations for binary mixtures of linear polybutadienes," Macromolecules 18, 2630-2643 (1986).

Utracki, L. A., in Multiphase Polymers: Blends and Ionomers, edited by L. A. Utracki and R. A. Weiss (American Chemical Society, Washington, DC, 1989), pp. 153-211. 
Valeur, B., L. Monnerie, and J. Rempp, "Physicochimie macromoleculaire-Insertion d'un fluorophore anthracenique dans une chaine de polystyrene. Etude des mouvements par inhibition et polarisation de fluorescence," C. R. Acad. Sci. Paris, Ser. C 270, 1009 1012 (1974).

Voigt-Martin, 1. G., K. H. Leister, R. Roseau, and R. Koningsveld, "Kinetics of Phase Separation in Polymer Blends for Deep Quenches," J. Polym. Sci.: Polym. Phys. 24, 723-751 (1986).

Watanabe, H. and T. Kotaka, "Viscoelastic and related properties of binary polymer blends," Chemtracts-Macromol. Chem. 2, 139-175 (1991).

Yang, H., H. Shibayama, R. S. Stein, N. Shimuzu, and T. Hashimoto, "Deuteration effects on the miscibility and phase separation kinetics of polymer blends," Macromolecules 19, 1667-1673 (1986). 